ÉGYPTE

monde arabe

\section{Égypte/Monde arabe}

19 | 2019

Les zabbâlîn, un objet surétudié ?

\title{
Travailleurs des tanneries, travailleurs des déchets du Caire. Paysages urbains singuliers, histoires de familles et savoir-faire professionnels
}

Tannery's workers and waste's workers in Cairo. Singular urban landscapes, family stories and professional skills

\section{Bénédicte Florin}

\section{Q OpenEdition \\ Journals}

Édition électronique

URL : https://journals.openedition.org/ema/4658

DOI : 10.4000/ema.4658

ISSN : 2090-7273

Éditeur

CEDEJ - Centre d'études et de documentation économiques juridiques et sociales

Édition imprimée

Date de publication : 1 janvier 2019

Pagination : 111-139

ISBN : 9782900956052

ISSN : $1110-5097$

\section{Référence électronique}

Bénédicte Florin, «Travailleurs des tanneries, travailleurs des déchets du Caire. Paysages urbains singuliers, histoires de familles et savoir-faire professionnels », Égypte/Monde arabe [En ligne], 19 | 2019, mis en ligne le 01 janvier 2021, consulté le 07 juillet 2022. URL : http://journals.openedition.org/ ema/4658; DOl : https://doi.org/10.4000/ema.4658 
TRAVAILLEURS DES TANNERIES,

TRAVAILLEURS DES DÉCHETS DU GAIRE.

PAYSAGES URBAINS SINGULIERS, HISTOIRES DE

FAMILLES ET SAVOIR-FAIRE PROFESSIONNELS

\section{RÉSUMÉ}

Au Caire, le quartier des Tanneries et les quartiers de chiffonniers constituent des espaces populaires et industrieux où vivent et travaillent de nombreuses familles employées à valoriser les "restes ", qu'il s'agisse des peaux ou des déchets. Singuliers, peu visibles et souvent menacés, ces micro-mondes se situent aux marges de la ville et leurs travailleurs et travailleuses se situent, également, aux marges de la société urbaine du fait de leurs activités considérées comme sales. Pourtant, la reconstitution de leurs itinéraires témoigne de leurs savoir-faire professionnels et de l'émergence d'une catégorie d'entrepreneurs qui a participé à la création progressive d'un « artisanat-industriel » . Celui-ci conserve, en partie, une dimension artisanale, informelle, familiale et communautaire, tout en empruntant, en partie, à la dimension industrielle ses outils, ses réseaux formels, sa concentration spatiale et sa force de travail. Non dénué de rapports de dépendance, sinon de domination, cet artisanat-industriel fonctionne de manière systémique sur la base d'une imbrication forte entre activités informelles et formelles et de circulations intenses dans la capitale. Déjà engagées, la démolition des Tanneries - en vue de leur relocalisation dans le désert - ainsi que celle du quartier des chiffonniers de Batn al-Baqara - en vue d'y favoriser la promotion immobilière privée - auront de graves conséquences économiques et sociales pour de nombreux travailleurs de ces quartiers. 


\section{INTRODUCTION ${ }^{1}$}

Dans le Vieux Caire - Masr el-Qadima -, 1000 tanneries, enregistrées à la Chambre des Tanneurs, ont été recensées afin d'être délocalisées à partir de 2017 dans le désert, auxquelles il faut ajouter sans doute bien davantage de petits ateliers sous-traitants de tannage, de fabrique de glu et gélatine, ou de récupération des poils et de la laine, non reconnus par la Chambre. Ces ateliers et leurs activités constituent l'essentiel du quartier des Tanneries ${ }^{2}$, espace populaire et industrieux où de nombreux travailleurs et leurs familles vivent. Au Caire, encore, sept quartiers de chiffonniers - zabbâlîn ${ }^{3}$ - rassemblent sans doute quelque 100000 personnes occupées à trier les déchets collectés en ville et à les recycler dans plus de 1000 ateliers informels. De nombreuses proximités permettent de rapprocher ces espaces de vie et d'activité : leurs paysages urbains les distinguant des autres quartiers ou des paysages industriels classiques, les origines et parcours des travailleurs ou, encore, le travail sur le " reste » que sont les peaux d'animaux ou les déchets avant leur transfiguration par le tannage ou le recyclage. Dans les deux cas, il est certain que les étapes initiales de cette transformation ressortissent d'un " sale boulot » (Hugues 1962, Lhuillier 2005), que le travail sur la matière brute est intense, que les effets sur la santé des travailleurs sont nocifs et toxiques et que l'ensemble du processus s'effectue en grande partie grâce à une main d'œuvre peu qualifiée et peu payée. Cependant, ces convergences doivent être nuancées : ainsi, le fait que le cuir, une fois tanné, "s'anoblit », ce qui est rarement le cas pour les déchets recyclés; ou encore, le fait que les tanneries, à la différence des quartiers de chiffonniers, peuvent devenir des lieux patrimoniaux et touristiques - à l'exemple de celles de Fès - bien que ce processus ne soit absolument pas engagé pour les tanneries centenaires du Caire, bien au contraire puisque leur démolition est d'ores et déjà engagée.

Il s'agit d'espaces de travail et d'habitat que l'on peut considérer comme des marges urbaines, que celles-ci soient relativement centrales ou périphériques, et de travailleurs qui se situent aux marges de la société urbaine, du fait de leurs activités sur le déchet. Pourtant cette position marginale est contrebalancée par

1 NB : Toutes les photos de cet article ont été prises par P. Garret que je remercie vivement ici (http://www.bab-el-louk.org/). Je précise encore qu'aucune photo n'a été prise sans l'accord des intéressés.

2 Je mets une majuscule à Tanneries lorsqu'il s'agit du quartier et une minuscule lorsqu'il s'agit de l'atelier où l'on tanne.

3 Le mot zabbâlîn (sing. zabbâl) dérive de zibbâla, la poubelle, et articule le mot " déchet » à ceux qui ont à faire avec, les chiffonniers. Les quartiers de ceux-ci peuvent parfois être appelés zarâyeb (sing. zerîba), littéralement " porcheries » (de zarrâba, porcher), terme très péjoratif lié à l'élevage des cochons se nourrissant des restes organiques. II me semble qu'aujourd'hui l'usage de ce terme tend à s'estomper. 
le fait que, chez les tanneurs ou les chiffonniers, les savoir-faire professionnels transmis d'une génération à l'autre, l'ingéniosité et l'évolution des techniques ainsi que les capacités d'investissement de certains se sont traduits par l'émergence d'une catégorie d'entrepreneurs, dès les années 1950-60 pour les tanneurs et, plus tardivement, pour les chiffonniers. Les trajectoires professionnelles des uns et des autres témoignent que s'est ainsi constitué progressivement un " artisanat-industriel ${ }^{4}$ qui conserve, en partie, une dimension artisanale tout en empruntant, en partie, à la dimension industrielle ses outils, sa concentration spatiale et sa force de travail. Cette évolution professionnelle ne concerne pas tous les protagonistes, loin de là, mais elle témoigne néanmoins de l'inclusion au secteur formel des activités des travailleurs et petits patrons qualifiés d'informels qui alimentent les fabriques ou usines formelles. Le propos n'est pas ici $d^{\prime}$ ouvrir un débat sur les imbrications entre les secteurs formel et informel ${ }^{5}$, mais davantage de montrer comment cette mutation s'est opérée pour ce qui concerne les travailleurs des tanneries et ceux des déchets.

En première partie de cet article, I'analyse en termes d'occupation du sol, d'un bâti évolutif et des imbrications entre lieu d'habitat et lieu de travail permet de mettre en perspective les quartiers des chiffonniers et celui des

4 En raison des différences de contextes historique, géographique et économique, j'ai préféré cette expression à celle de " proto-industrie » (Mendels 1969) qui, dans sa définition première, s'applique aux ateliers de paysans, en milieu rural, qui alimentaient en produits d'artisanat local les manufactures nées de la révolution industrielle. "Artisanat industriel » renvoie ici à une organisation du travail familiale et communautaire, aux capitaux investis relativement limités, à la forte présence de l'informel, aux procédés de fabrication en général artisanaux (hormis quelques exceptions). La dimension " industrielle » s'explique pourtant par le fait que ces activités sont fortement reliées aux réseaux industriels et commerciaux du recyclage et du tannage, à un chiffre d'affaires global — impossible à évaluer, sauf méconnaissance de ma part sans doute important et, enfin, du fait de la concentration de ces multiples fabriques. Notons que j'ai eu la chance de visiter, en 1996, la grande usine publique de tannage, située à Basatîn. L'usine, dont les machines-outils furent importées d'URSS dans les années 1960, fonctionnait déjà au ralenti et la délocalisation en 1995 des abattoirs à proximité n'a pas empêché sa fermeture.

5 Il serait trop long ici de référer aux nombreux travaux portant sur les formes d'inclusion et de dépendance du secteur informel de la récupération des déchets au secteur formel du recyclage. Pour notre part, nous avons abordé cette question dans «Vivre des déchets » (Cirelli, Florin 2015) en l'inscrivant dans les apports théoriques d'autres chercheurs (se reporter notamment aux autres articles de cet ouvrage ainsi qu'aux bibliographies afférentes). Enfin, nous ouvrons en conclusion de cet article le débat via les effets sur le secteur informel des projets de démolition de certains de ces quartiers. 
Tanneries : pour les travailleurs ${ }^{6}$ des tanneries et des déchets, la fabrication de leurs espaces de vie se superpose aux espaces d'activité. De façon plus générale, les marqueurs spatiaux, définissant des paysages urbains spécifiques, sont étroitement liés aux marqueurs sociaux et professionnels qui renvoient à des représentations souvent convergentes entre travailleurs des déchets et des tanneries quant à leurs quartiers respectifs. Leur clôture spatiale, leur localisation dans les failles et interstices de la ville, la nature des activités en font des marges, même lorsque leur localisation est quasi-centrale. Ces caractéristiques expliquent leurs déplacements forcés par le passé et la délocalisation du quartier des Tanneries, matérialisée par de premières démolitions en 2017, puis stoppée en raison de l'opposition d'une partie des petits patrons d'ateliers.

En deuxième partie de cet article, la reconstitution des itinéraires résidentiels et professionnels de ces travailleurs montre qu'il s'agit majoritairement de migrants ruraux de Haute-Égypte, mus par la pauvreté à partir des années 1930 pour les chiffonniers et avec un «pic » dans les années 1950 pour les travailleurs des tanneries implantées sur ce site à partir de la fin du $\mathrm{XIX}^{\mathrm{e}}$ siècle. Ces histoires résidentielles mouvementées, marquées par l'incertitude ${ }^{7}$ de l'arrivée en ville, reposent sur l'entremise de la famille élargie et de la communauté qui connaissent les opportunités de travail et de logement au Caire, donnant ainsi un sens aux trajectoires des travailleurs. Celles-ci diffèrent pour les patrons des grandes tanneries car une partie d'entre eux était d'origine étrangère tandis que les autres sont Cairotes depuis plusieurs générations. Par la suite, les parcours professionnels peuvent diverger en fonction de la nature des activités de chacun et de l'ascension professionnelle réalisée par certains et non par d'autres ainsi que le montreront les généalogies de tanneurs et de chiffonniers. Mais, de façon générale - et qu'il s'agisse des activités de tannage ou de recyclage -, la modernisation des machines-outils, l'innovation technique, I'intégration dans des circuits commerciaux locaux, nationaux et internationaux sont la preuve de l'émergence de l'artisanat-industriel présenté ci-dessus qui n'empêche pas les très nombreuses petites activités employant une importante main d'œuvre mal-payée. Le mode de fonctionnement de ces activités est systémique et se traduit par une sous-traitance forte dont dépendent les petits ateliers de tannage, fabriques de gélatine mais aussi, pour les zabbâlîn, les trieurs et trieuses de déchets par exemple. À une autre échelle, les activités de recyclage des

6 J'emprunte à D. Corteel et S. Le Lay le terme « travailleurs » car « ouvriers » correspond mal aux nombreux travailleurs informels (Corteel, Le Lay 2011), hormis lorsque les personnes rencontrées utilisent ce terme, notamment dans le cas des tanneries légales. Je distingue également les " travailleurs » des " patrons ».

7 Dans un tout autre contexte, Giovanni Levi montre que "l'incertitude » - la précarité de la vie - est tout à fait intériorisée par les acteurs qui mettent en œuvre toutes sortes de stratégies d'alliances familiales, matrimoniales ou politiques dans le but de maitriser ou au moins de réduire cette incertitude (Levi 1985). 


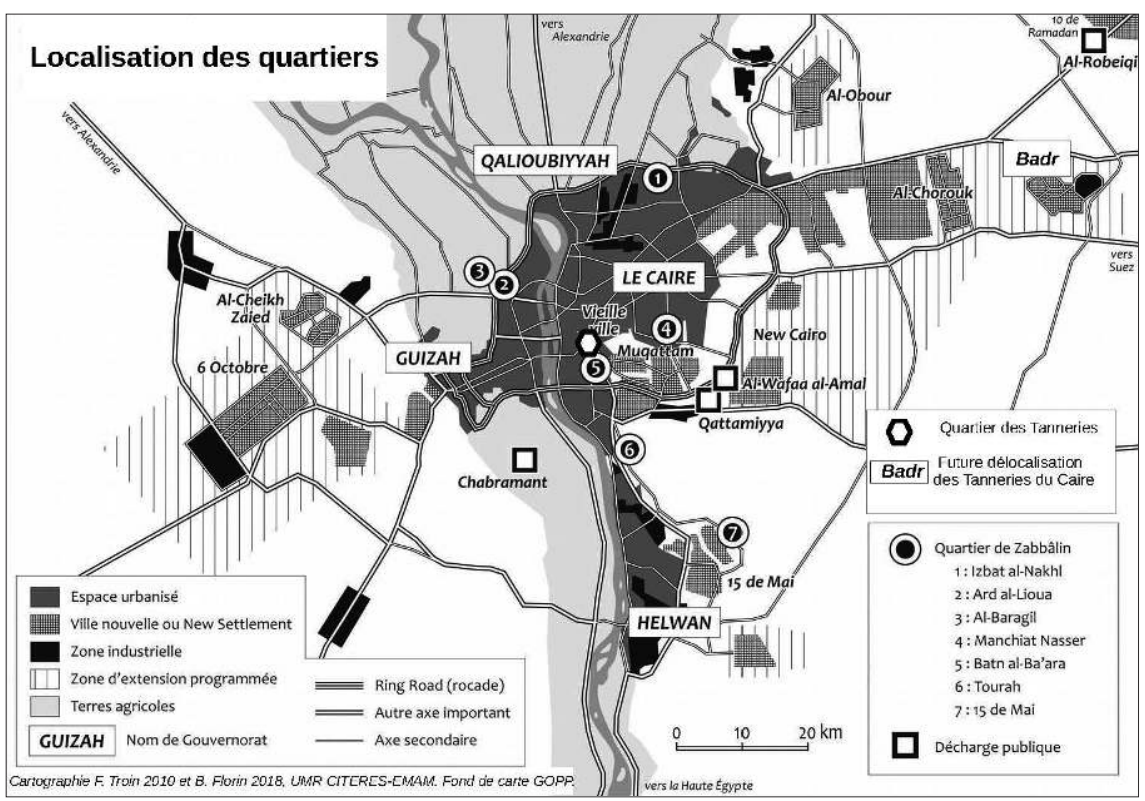

Figure 1 : Carte de localisation des quartiers

chiffonniers sont étroitement liées aux usines du Caire et à des filières commerciales étrangères. Engagée en 2016, la délocalisation des tanneries dans le quartier industriel de Robeki (ville nouvelle de Badr) à 80 km du Caire constituera très certainement une rupture considérable de ces synergies ${ }^{8}$.

8 Sur le plan méthodologique, mon travail d'entretiens qualitatifs sur les itinéraires résidentiels et professionnels d'une quarantaine d'habitants-travailleurs des Tanneries ainsi que sur l'histoire et l'activité d'une dizaine de patrons tanneurs a débuté entre 1993 et 1995, dans le cadre de ma thèse de doctorat de géographie (Florin 1999). L'extrême bienveillance des habitants, la richesse de leurs propos, la connaissance dès cette époque du projet de délocalisation qui touche la zone et donc de leur dispersion probable ont contribué à mon attachement profond pour ce lieu et les gens qui y vivent. Par la suite, chaque retour au Caire a été l'occasion de visites, de nouvelles observations et discussions. J'ai initié mes recherches sur les chiffonniers à partir de 2007, avec des retours en 2009, 2010 et 2012. La plupart de mes entretiens se sont déroulés à Manshiyyat Nâsir et, dans une moindre mesure, à 'Ard al-Lewa, Batn al-Baqara et Tourah. En février 2017, un séjour de 10 jours m'a permis de mener de nouveaux entretiens, axés sur le travail, aux Tanneries, à Manshiyyat Nâsir et Batn al-Baqara. Ces entretiens de 2017 ont été menés en partie seule, en partie avec un étudiant égyptien, Karim Selim, qui m’a aidée pour les traductions ainsi qu'avec Pascal Garret, photographe, qui a réalisé de nombreux portraits de travailleurs. Enfin, cet article présente un itinéraire de recherche m'ayant menée des Tanneries aux chiffonniers. 


\section{QUARTIER DES TANNERIES, QUARTIERS DES CHIFFONNIERS :} DES ESPACES SINGULIERS, INVISIBLES ET MENACÉS

\section{Paysages urbains, paysage du travail, paysage sociaux}

Bien que situées au sud du centre-ville, le Cairote ou l'étranger, connaît peu et pénètre difficilement dans les Tanneries, el-Madabegh. Au nord, un imposant aqueduc $^{9}$, laisse seulement deux passages par lesquels on distingue les fragments d'un espace d'activités intenses et d'habitat précaire pourtant vaste $d^{\prime}$ 'une trentaine $d^{\prime}$ hectares. La première entrée ouvre sur la rue Fustât, aujourd'hui obstruée par les activités de tannage. On peut y entrevoir des hommes et des adolescents affairés, des charretiers dont les carrioles sont surchargées de peaux suintantes ou déjà traitées ainsi que les cadres en bois où sont cloutées d'autres peaux qui sèchent. La seconde entrée correspond à la voie ferrée, ensevelie aujourd'hui, reliant le centre-ville aux banlieues sud et qui passait par les abattoirs fermés en $1995^{10}$. Les voitures, camions ou pick-up des tanneurs et les charrettes sont les seuls véhicules à se frayer un chemin malaisé par ces deux voies. De hautes claies en bois à ciel ouvert surplombent I'aqueduc : y sèchent les plaquettes de gélatine jaune dont la section carrée correspond aux bidons d'huile récupérés et utilisés pour la fondre. Cette gélatine dorée, translucide sous le soleil, est fabriquée à partir des rebuts organiques raclés sur les peaux et sert de liant pour l'industrie agro-alimentaire. D'autres bidons posés à même le sol contiennent la glu noire, utilisée comme colle. Même la « vitrine » des Tanneries qui prend la forme de magasins d'exposition, reste peu visible car masquée par l'Aqueduc : on y voit des peaux suspendues, dont des peaux de zèbres, et l'on peut y croiser un taxidermiste qui nous présente un crocodile du Nil et une peau de tigre avec sa tête...

Respectivement à l'est et au sud, le grand ensemble de logement social 'Ayn el-Sira ainsi qu'une cité de relogement d'urgence, très densifiée aujourd'hui, les Kiosques - Koshk - Abû el Su'ûd, enclosent le quartier des Tanneries. À l'ouest, une grande fabrique de recyclage d'os, détruite fin 2016, fermait le quartier. Pourtant, I'autonomie de ces différents espaces est relative : les tanneries emploient depuis longtemps des hommes des quartiers voisins qui ont trouvé à s'y loger et, de même, les fabriques de glu et gélatine se sont implantées sur le pourtour nord de la cité 'Ayn el Sira. Dans chacun de ces quartiers limitrophes,

9 «L'aqueduc, bâti sur des arcs reposant sur des piliers, se dirigeait vers l'est en reprenant le tracé de l'aqueduc de Nâsir, rattrapait le mur de Saladin, obliquait ensuite vers le nord-est et aboutissait à l'Hippodrome et à la Citadelle. L'ouvrage (...) fut édifiée de mai 1507 à octobre 1508 ». (Raymond 1993 : 184).

10 Héritage de cette activité, de nombreux bouchers, couteliers et vendeurs de billots sont toujours présents à l'endroit des anciens abattoirs dont les peaux étaient donc vendues aux tanneries tout proches. 


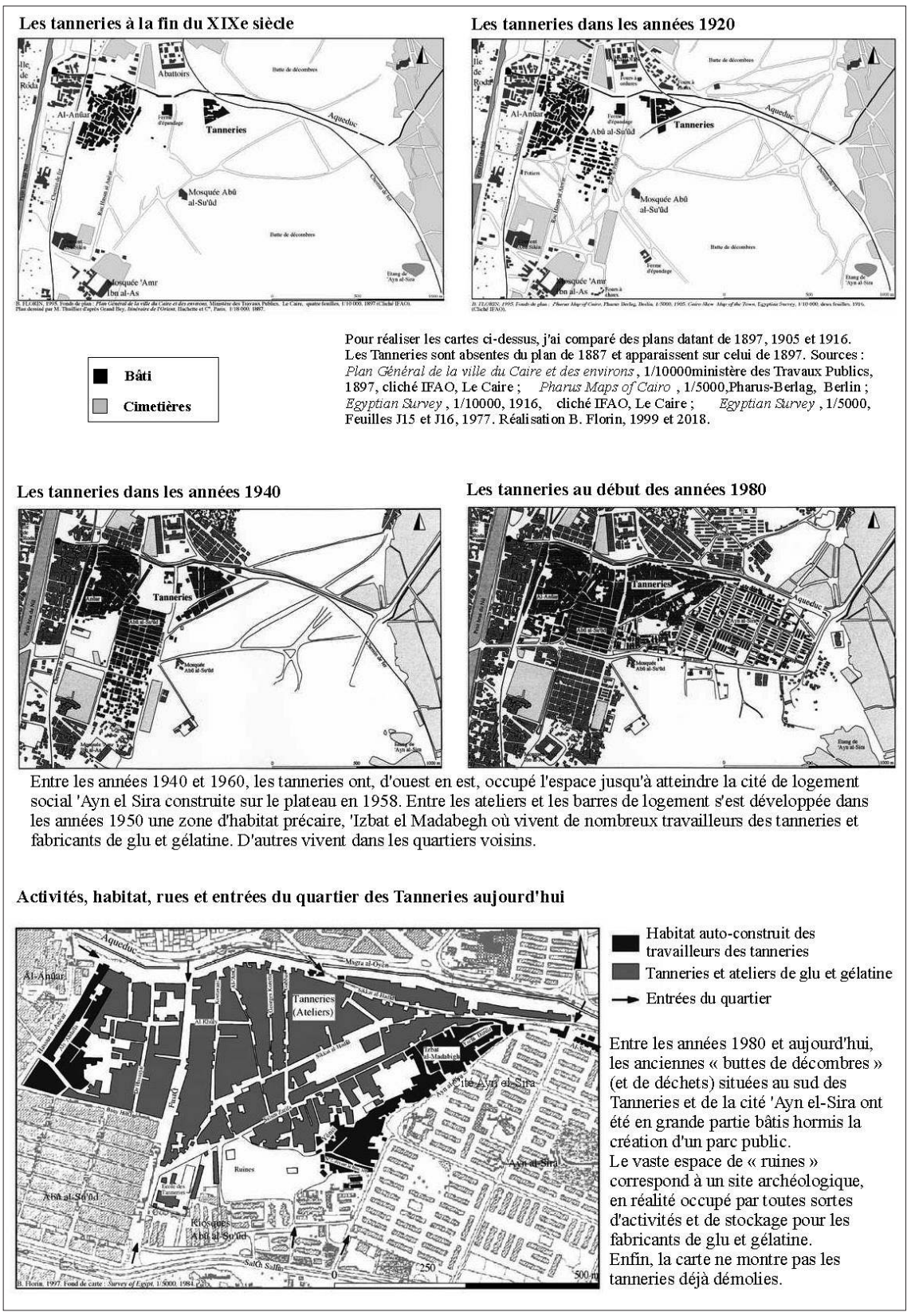

Figure 2 : Cartes des Tanneries à la fin du XIXe s., en 1920, 1940, 1980 et activités, habitat, entrées et rues du quartier aujourd'hui 
de nombreuses petites activités ainsi que des commerces de produits chimiques nécessaires au traitement des peaux annoncent l'activité de tannage.

À la suite de la création en 1890 des abattoirs « modernes » du Caire, les tanneries s'implantent sur le site à partir de la fin du XIX $x^{\mathrm{e}}$ siècle, dont trois ateliers appartenant à Hussein al-Gabbas, donnant son nom à la rue éponyme. D'après Mahmûd al-Gabbas, arrière-petit-fils de Hussein et tanneur lui-même, I'attribution de ces terrains aurait servi de dédommagement à la délocalisation des tanneries ${ }^{11}$ de Bâb al-Lûq, au centre-ville, et de Masr al-Atiqa, au sud du Caire, vers Masr al-Qadîma, leur arrondissement actuel (Raymond, 1977 : 195). Le site, à la topographie accidentée, regorge d'eau souterraine. À partir des années 1950, la multiplication des ateliers de tannage nécessite davantage de main d'œuvre et une population masculine, pauvre, originaire de HauteÉgypte, trouve à s'y faire embaucher tout en construisant des 'ichach à 'Izbit al-Madabegh ${ }^{12}$, un terrain public qu'ils squattent. Nous reviendrons ci-dessous sur les itinéraires des travailleurs des tanneries, mais les plus âgés aujourd'hui se souviennent que c'était alors la " montagne »-gabal -, un endroit inhospitalier et sauvage où il y avait « des cochons et des prostituées » (Ali, entretien du 10/09/1995), des afarît (mauvais esprits), où I'on était isolé, où l'on avait peur, si bien que « les tanneurs enfermaient les ouvriers dans leurs ateliers pour les rassurer »(Chaûqi, entretien du 14/09/1995). Le politiste Alain Roussillon rapporte ainsi la perception, dans les années 1940, de la zone des abattoirs et des tanneries :

« Les collines de Zaynhom sont un haut lieu de la prostitution (baghâ'), exercée en plein air par des femmes dont les visages pâles et émaciés et les haillons disent assez la condition misérable (...) En leur interdisant d'opérer dans d'autres parties de la ville, [la police] refoule ces femmes vers ces contrées sinistrées et contribue encore à aggraver le sort des ouvriers des tanneries qui constituent l'essentiel de leur clientèle» (Roussillon 1996 : 27).

11 Antoine-Barthélémy Clot-Bey, médecin du vice-roi d'Égypte Méhemet Ali, recense en 1840 les corporations égyptiennes et écrit à propos des tanneries : "Les tanneries forment une industrie assez considérable. Les Égyptiens y suivent des procédés qui leur sont particuliers, ils tannent les peaux de vaches, de buffles, de moutons, de chèvres, etc. Ils réussissent très bien dans la préparation du maroquin qui n'est autre que la peau de chèvre teinte (...) », (1840:290). André Raymond précise que, avant la période ottomane (1517), les tanneries étaient situées aux portes de la vieille ville du Caire, à 300 mètres de Bâb Zwaïla précisément, avant d'être déplacées vers 1600 en raison des odeurs nauséabondes qu'elles dégageaient et de la croissance urbaine (Raymond $1990: 40$ ).

12 Le mot 'ech (plur. 'echach) vient de 'ush (nid) et signifie bicoque, taudis (Ilbert 1989 : 280). 'Izba (plur. 'izab) désigne un quartier pauvre et précaire. Le terme est péjoratif. 


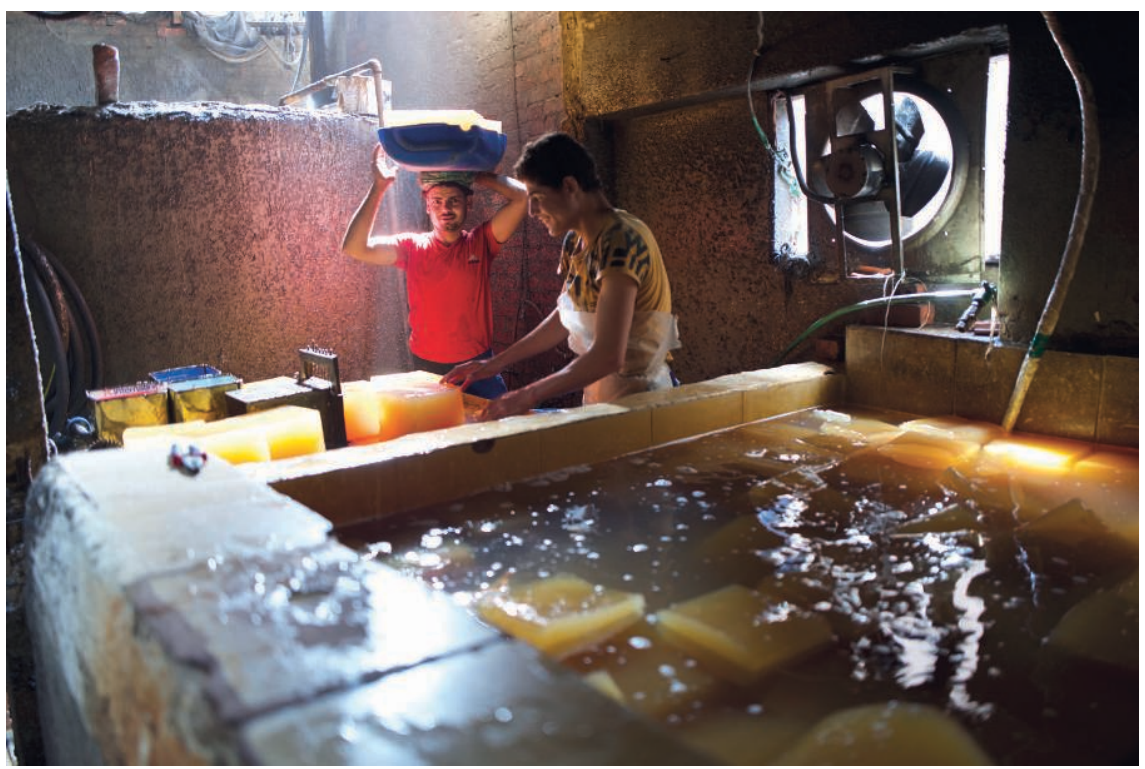

Figure 3 : Un atelier de gélatine. Dans cet atelier « informel», les résidus organiques sont raclés sur les peaux, puis portés à ébullition dans de grands chaudrons. Le liquide est ensuite versé dans des bidons mis à refroidir. Ici, deux frères découpent les blocs en plaquettes qui sécheront sur les hautes claies en bois qui dominent le quartier (photo Pascal Garret, Le Caire, 2017)

Pourtant, en 1933, se construit un Institut (privé) professionnel du cuir et de la chaussure devenu public en 1957 et démoli en 2016. Le bâtiment, que j'ai visité en 1996, affichait dans son hall d'entrée les tableaux d'honneur des tanneurs diplômés de I'Institut. À partir des années 1950-60, de nombreuses activités dérivées et informelles se sont multipliées : petits ateliers de tannage, fabriques de glu et gélatine, magasins de produits chimiques, ateliers des récupérateurs de laine, petits lieux de restauration, vendeurs de thé et café, etc. Alors sans électricité, les tanneurs utilisaient des "babûr diesel », moteurs alimentés en essence et en huile de vidange qui faisaient tourner les baramil, terme sans doute dérivé de baril, à savoir des gros tonneaux de bois pour les plus anciens, en métal pour les récents qui tournent sur eux-mêmes et dans lesquels on lave et traite les peaux.

Dans les années 1990, l'organisation de l'activité est la suivante : peu de tanneurs sont propriétaires de leur terrain mais beaucoup ont une activité formelle inscrite à la Chambre des Tanneurs. Ces derniers bénéficient d'un contrat de location du sol - hikr - qui concède l'usage du terrain en échange d'une somme payable annuellement au Gouvernorat et établie en fonction de la surface occupée. C'est également ce hikr qui, après plusieurs confrontations violentes entre les premiers occupants et les pouvoirs publics, est concédé aux travailleurs des tanneries qui squattent 'Izbat el Madabegh pour y construire leurs habitations de fortune (Florin, 1999). 


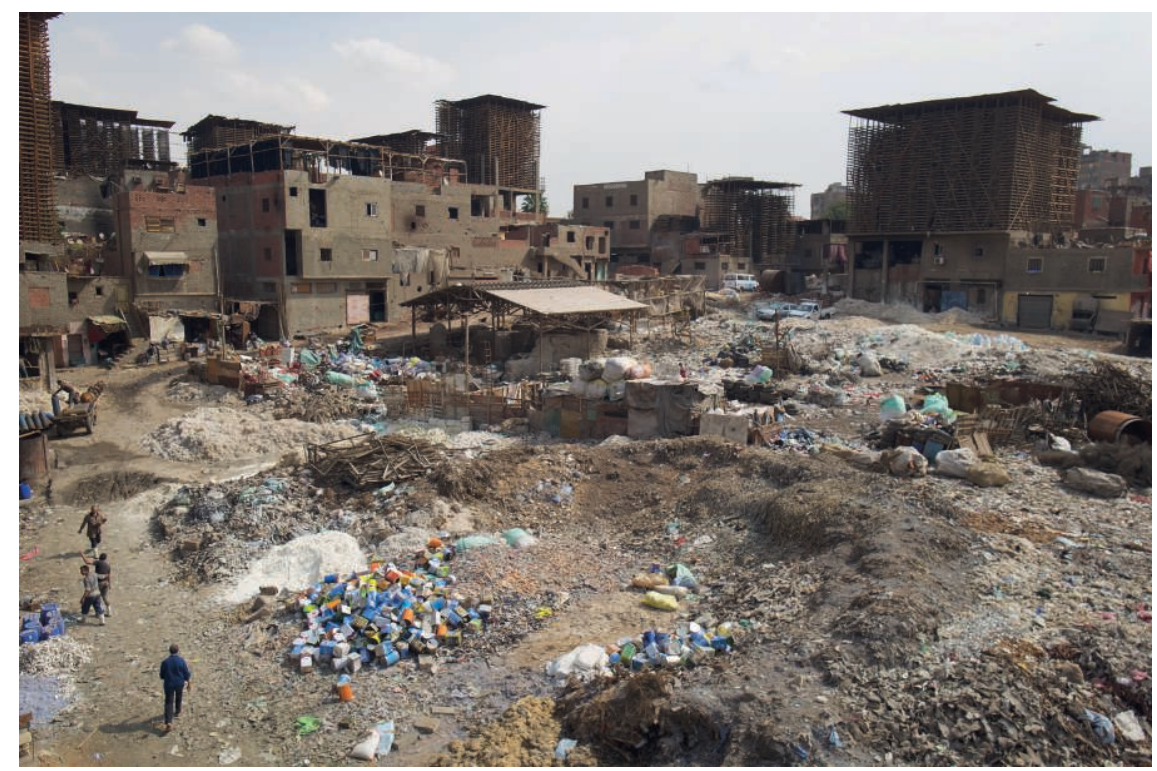

Figure 4 : Vue d'ensemble des Tanneries. Les ateliers de glu et de gélatine repérables par la présence des bidons bleus et des séchoirs en bois (photo Pascal Garret, Le Caire, 2017)

Les tanneries et activités liées ont une force visuelle et olfactive participant à construire un paysage spécifique constitué de bâtiments hétéroclites dont les plus anciens sont bas et en pierre et dont les plus modernes, hauts de 5 ou 6 étages, sont en béton armé pour soutenir les lourdes machines modernes. S’y ajoutent les émanations et fumées, les canalisations à ciel ouvert où coule une eau très bleue, chargée de chrome et $d^{\prime}$ autres produits chimiques ${ }^{13}$, le bruit sourd des baramil qui tournent ou le cliquetis des clous, les allers et venues des porteurs de peaux et des carrioles tractées par des chevaux, etc. Le tissu est dense, les rues étroites et les impasses nombreuses. L'imbrication entre activités et habitat des travailleurs - car les patrons résident ailleurs - constitue un lien fondamental entre les habitants et «leur quartier» et contribue ainsi de manière puissante au sentiment d'appartenance à un lieu et à un travail. Et, de la même façon qu'à Manshiyyat Nâsir, le plus grand des quartiers de chiffonniers, tout le monde ou presque, aux Tanneries, se connaît ${ }^{14}$.

13 En surface dans le quartier, cette eau est ensuite canalisée par des égouts jusqu'au Nil. La pollution qui en découle est l'un des arguments avancé pour la délocalisation des tanneries.

14 C'est en montrant à des travailleurs des photos de leurs collègues, parfois éloignés du lieu où j'étais, que ces interconnaissances m'ont semblé évidentes. 


\section{DES ESPACES TOUJOURS MENACÉS : EXPULSIONS, DÉMOLITIONS ET DÉLOCALISATIONS}

L'une des convergences entre les chiffonniers et habitants-travailleurs des Tanneries est le fait d'avoir vu leurs habitations détruites à plusieurs reprises entre les années 1950 et 1979. Mais, à la différence des chiffonniers qui seront souvent déplacés, les gens des Tanneries reviennent construire sur place leurs bicoques jusqu'à l'obtention du hikr, en 1979 : "On a construit la nuit, ils [la police] ont cassé le jour. Ils ont cassé quatre fois la maison. On est restés comme ça très longtemps, avant d'avoir le hikr. Ensuite, on a construit une pièce en briques et en bois. C'était une montagne et on a aplani [le terrain] On a construit nous-mêmes, pièce par pièce. » (Fangaria, arrivée aux Tanneries en 1964, entretien du 6/04/1994).

Les campements de fortune des chiffonniers, quant à eux, sont détruits à sept reprises par les autorités, entre 1950 et 1970. Les expulsions s'effectuent brutalement au petit matin, en présence de la police et à l'aide de bulldozers : les habitants ont juste le temps de prendre l'essentiel de leurs affaires, puis de trouver un autre lieu discret qu'ils pourront squatter. Initialement installés à Abû Wafi', aux confins nord du Caire et à côté de l'emplacement où les wahiyya $^{15}$ - oasiens - faisaient sécher les déchets, les premiers migrants déménagent à al Basrawi (rive ouest du Nil). Repoussés par la croissance urbaine, par les autorités, mais aussi rejetés par les habitants en raison des déchets et surtout des cochons ${ }^{16}$, ils repartent ensuite à Mattariyyah (au nord), puis, au-delà encore, à 'Izbat al Nakhl ${ }^{17}$. Un certain nombre de familles reste dans ce bidonville, qui sera rapidement entouré d'immeubles. Pour de nombreux autres chiffonniers, la dernière étape, en 1969, est le pied du Muqattam, falaise qui borde l'est du Caire, lieu inhospitalier s'il en est car à l'époque désertique et dominant la Cité des Morts ${ }^{18}$. Dans ce qui deviendra le grand quartier de Manshiyyat Nâsir, les

15 Ce groupe est assez mal connu : il s'agit de musulmans originaires des oasis du désert libyque. Ces wahiya menaient leurs affaires dans des cafés rue Clot Bey, dans le quartier central de Bâb el-Bahr. Dans les quartiers populaires, les cafés peuvent être des lieux d'embauche où les employeurs savent trouver une main d'œuvre disponible et parfois spécialisée dans certaines professions, notamment les arzuqi ou journaliers.

16 La plupart des sources, orales ou écrites, s'accorde à dire que les cochons, animaux impurs dans le monde musulman, ont été élevés par les chrétiens coptes pour la consommation des étrangers, les Britanniques en particulier. Notons ici que les chiffonniers musulmans élèvent également des porcs [Florin, 2011].

17 Se reporter à la carte de localisation des quartiers de chiffonniers présentée en fin $\mathrm{d}^{\prime}$ Introduction.

18 En Égypte, les espaces désertiques ou les cimetières suscitent le rejet : ce sont des lieux dangereux, où errent notamment les mauvais esprits et animaux sauvages. 
zabbâlîn vont reconstruire leurs cabanes et porcheries. D'autres familles n'ont pas déménagé à Manshiyyat Nâsir, mais se sont installées à la lisère ouest de la métropole, formant deux plus petits quartiers de chiffonniers, Baraguil et 'Ard al Lewa, aujourd'hui séparés par un large périphérique.

S'ajoute encore le petit quartier de Batn al Baqara, le Ventre de la Vache, ancienne carrière où les bouchers des abattoirs venaient jeter les entrailles invendables des animaux. Coptes ou musulmans, les habitants de Batn al Baqara considèrent leur quartier comme « le berceau des chiffonniers » : datant sans doute des années 1940, sa localisation s'explique par la proximité de la décharge de 'Ayn al Sira, aujourd'hui convertie en parc.

Au sud du Caire, le quartier de Tourah a un statut un peu spécifique puisqu'il s'agit d'un ancien bidonville, datant de 1975, démoli en 1989 et remplacé par des bâtiments abritant des familles de chiffonniers, pour moitié musulmanes. Enfin, plus récemment s'est constitué, plus au sud, un autre petit quartier, le plus pauvre de tous, sans eau, ni électricité : les chiffonniers y collectent les déchets des quartiers aisés de la banlieue sud mais aussi ceux de la grande décharge de Qattamiyyah où ils vont ramasser illégalement les ordures.

Le travail de tri des déchets, revente des matériaux à des commerçants et l'élevage des porcs constituent l'essentiel de l'activité des chiffonniers de Manshiyyat Nâsir jusqu'aux années 1980. Pour la plupart d'entre eux, la rupture essentielle correspond à l'obtention du hikr qui impulse la transformation du bidonville initial en immeubles de plusieurs étages ${ }^{19}$ et surtout à l'arrivée de l'électricité en 1985 qui permet l'essor de nombreuses activités de recyclage techniquement de plus en plus perfectionnées jusqu'à la quasi-industrie du recyclage actuelle. Pourtant, tous ne disposent pas de cette reconnaissance foncière que constitue le hikr, notamment ceux qui ont construit des immeubles ou de nouveaux ateliers sur les terrains encore vacants à la suite de la révolution de 2011, dont I'un des effets notoires fut une intense activité de construction non réglementée dans toute la ville...

Dans les immeubles de Manshiyyat Nâsir, la superposition de l'espace de travail et de l'espace de vie est littérale puisque dans les rues et en rez-de-chaussée s'effectuent le tri des déchets et le travail de recyclage, dans les étages se trouvent les appartements alors que les toits-terrasses servent de lieux de stockage des matériaux imputrescibles. Par contre, le quartier de Batn al-Baqara est de construction beaucoup plus précaire : les petites habitations en pierre servent aussi de logements sans eau courante et aux branchements électriques illégaux. On y trouve de nombreux petits ateliers de recyclage (broyage du plastique, compactage du carton) qui fonctionnent grâce à des groupes électrogènes.

19 Ma première visite dans le quartier date de 1993, année où les immeubles de 5 et 6 étages étaient déjà nombreux. À cette date, les cochons des zabbâlîn vaquaient en toute liberté dans les rues du quartier alors que par la suite ils furent parqués dans des enclos ou cours fermés. 


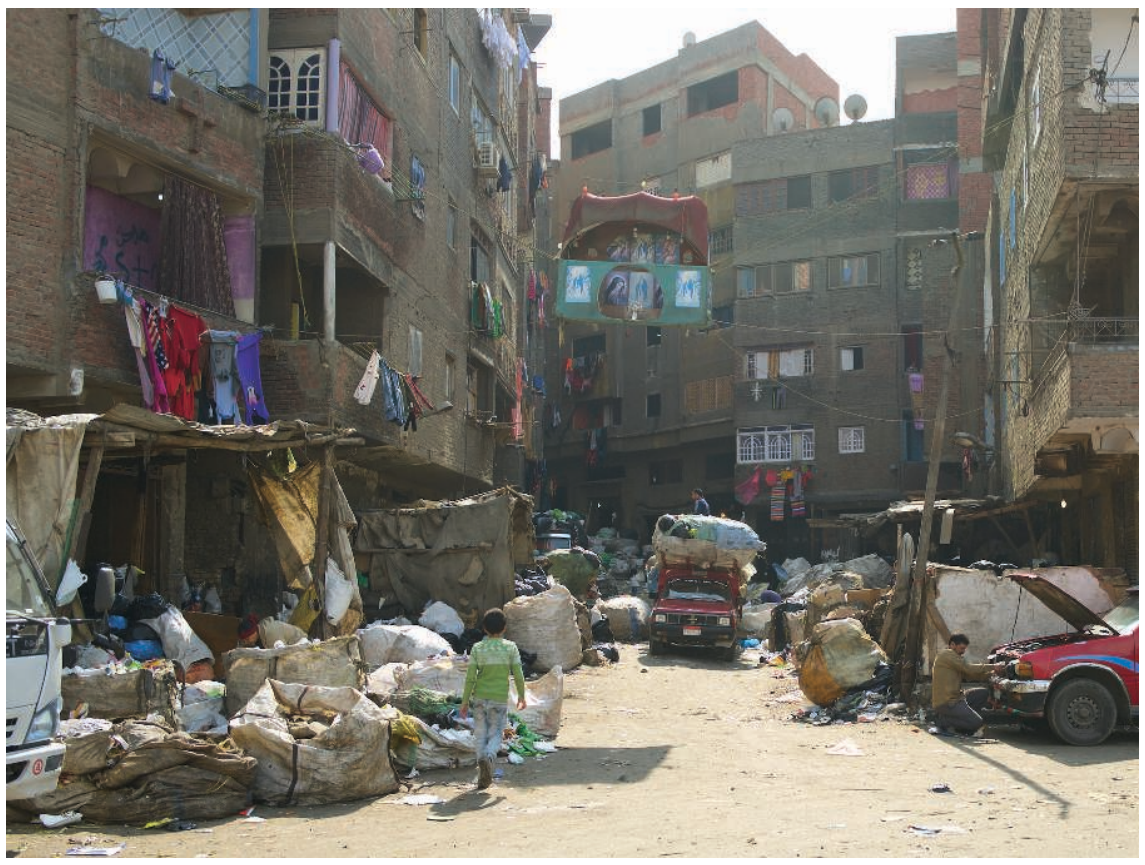

Figure 5 : Une rue de Manshiyyat Nâsir. Le contenu des poubelles collectées en ville est trié dans les rues ou en rez-de-chaussée des immeubles où se trouvent également les ateliers. Les appartements se situent dans les étages et les toits-terrasses servent souvent d'espace de stockage pour des déchets secs. Un autel copte est suspendu témoignant de l'identité religieuse du quartier (photo Pascal Garret, Le Caire, 2017)

Quoi qu'il en soit de leurs différences, les quartiers de chiffonniers, à l'instar des Tanneries, sont marqués par leur invisibilité : peu accessibles, il s'agit d'espaces que l'on ne traverse pas, où l'on ne se rend pas a priori. Ainsi, peu de Cairotes savent où réside le chiffonnier qui vient chercher leur poubelle au petit matin et, paradoxalement, ce sont davantage les touristes et les pèlerins étrangers qui connaissent les églises de Manshiyyat Nâsir, creusées dans la falaise du Muqattam. Dans ce même quartier, seules quelques voies sont vraiment carrossables et empruntées par un va-et-vient incessant de charrettes, pick-up, camions et depuis quelques années par les touks-touks ${ }^{20}$, alors qu'y travaillent sans doute quelque 60000 personnes $^{21}$. Quant au quartier de Batn al-Baqara, il s'agit d'un espace en cul-de-sac, situé dans une dépression topographique, et ce qui s'y déroule est vraiment indécelable de l'extérieur sauf si l'on porte

20 D’abord relégués dans les périphéries populaires les touk-touk (autorickshaw) sont nombreux à Manshiyyat Nâsir; plus récemment, ils ont envahi la vieille ville et des quartiers populaires plus centraux.

21 De la même façon que pour les travailleurs des tanneries, les zabbâlîn sont difficiles à dénombrer. 
attention aux véhicules chargés de matériaux récupérés et à la présence d'un pont-bascule ${ }^{22}$. Or, c'est aussi un lieu d'activité pour environ 5000 personnes.

Les zabbâlîn sont soudés par la force communautaire - car majoritairement coptes $^{23}$ et de même origine géographique, le Sa'îd -, par l'endogamie et par le travail avec les déchets. Cette superposition des dimensions identitaires - religieuse, individuelle et collective, sociale et professionnelle - construit la communauté, voire une forme corporative ${ }^{24}$, I'ancre dans une histoire et dans I'espace. Elle est constitutive des façons de travailler dans la discrétion et des modes d'habiter à la marge.

Ceci explique que leurs espaces de vie et de travail soient situés aux confins de la ville. Certes, au Caire, bien d'autres quartiers pauvres se sont élaborés selon les mêmes processus de squat et ne disposent ni d'eau courante, ni des services élémentaires comme à Batn al-Baqara. Mais, la marginalisation réside dans la double peine spécifique qui pèse sur les quartiers de chiffonniers marqués du sceau de l'impur du fait du côtoiement des porcs et des ordures. Les chiffonniers sont eux-mêmes également producteurs de cette marginalisation qu'ils ont intégrée dans leurs pratiques professionnelles, notamment en collectant les déchets discrètement. Aussi, l'invisibilité spatiale et sociale s'articuleà leur invisibilité professionnelle et ce jusqu'aux années 2000 lors desquelles les réformes du système de gestion des déchets les mettent sur le devant de la scène publique (Debout, Florin 2011; Desvaux, Furniss 2015; Florin 2015).

Aux Tanneries, le travail sur les peaux, que l'on peut aussi considérer comme un rebut, est difficile, sale et polluant, justifiant la situation de marge spatiale et sociale. De plus, les habitants et travailleurs des Tanneries ont mauvaise réputation : les trafics de haschich dans le quartier, supposés ou avérés selon les habitants eux-mêmes, les liens avec les bouchers (qui ont eux-mêmes mauvaise réputation) et la nature du travail expliquant cette disgrâce.

Les menaces de délocalisation sont aujourd'hui plus que tangibles puisque le projet de déplacement des tanneries, vieux de trente ans, est engagé fin 2016

22 Le pont-bascule sert à peser les pick-up et charrettes vides et pleins, afin d'obtenir par soustraction la quantité de matériaux collectés et d'en calculer le prix.

23 Les coptes représentent une minorité régulièrement en butte à des discriminations. Cette dimension religieuse joue un rôle majeur dans le sentiment identitaire constitutif de la communauté des chiffonniers mais aussi dans ses relations avec la bourgeoisie copte qui y intervient par des actions de bienfaisance.

24 Les corporations de métiers ont fait l'objet de nombreuses attentions et on pourra notamment se reporter à I'article d'André Raymond sur « Les transformations des corporations de métiers au Caire du $\mathrm{xVIII}^{\mathrm{e}}$ au $\mathrm{xIx}^{\mathrm{e}}$ siècle » expliquant les raisons de leur disparition en tant que telles (modernisation de l'économie et de l'administration) à la fin du xIx ${ }^{\mathrm{e}}$ siècle. Ici, nous entendons par « forme corporative », une organisation structurée en interne et tentant de défendre ses droits par la voix de ses différents porte-paroles qui ont acquis une autorité liée à leur position professionnelle ou religieuse. 


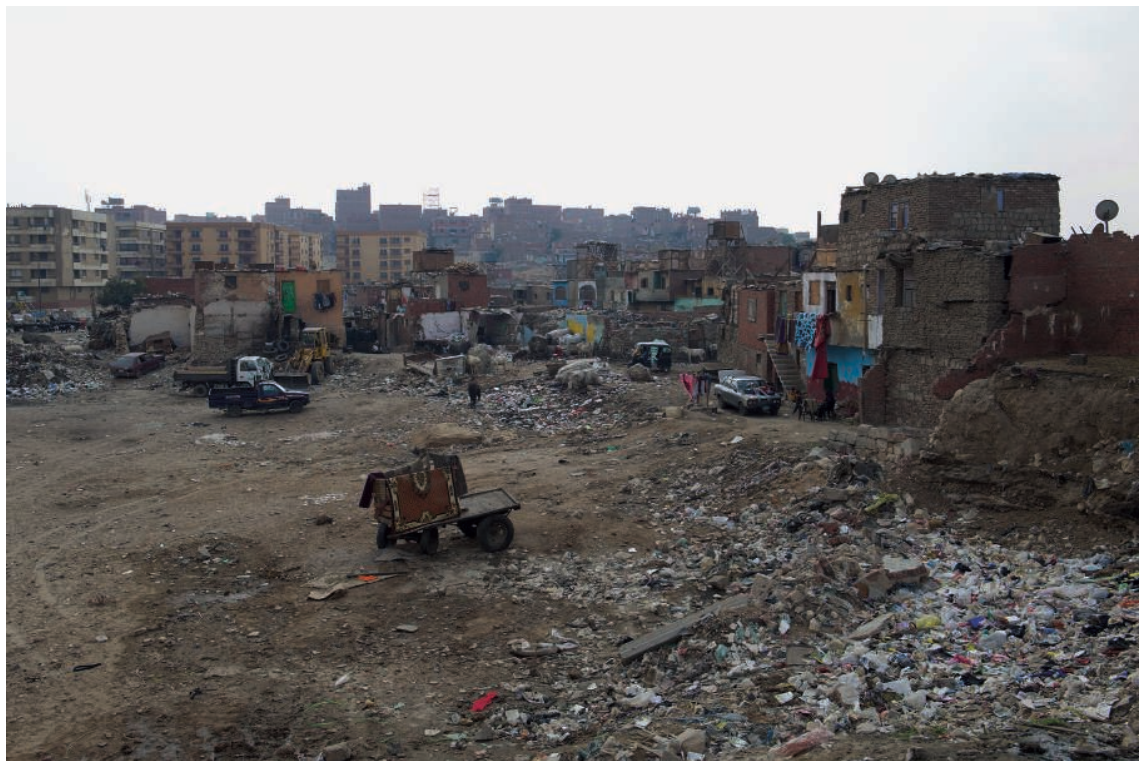

Figure 6 : Vue d'ensemble de Batn al-Baqara (2017). Cette vue montre, à gauche, les immeubles récemment construits à la place d'habitations démolies. L'activité de collecte se décèle par la présence des pick-up, charrettes et ânes tandis que les ateliers de tri et recyclage se situent à I'intérieur du quartier. Au fond, on aperçoit l'extrémité nord du quartier Istabl Antar (photo Pascal Garret, Le Caire, 2017)

avec la destruction des premières fabriques et habitations. Il en est de même à Batn al-Baqara dont les habitants-chiffonniers, déjà violemment menacés par les promoteurs aidés de la police en 2009, avaient bénéficié d'une sorte de sursis après la révolution de 2011. Mais, au début de l'année 2017, la démolition du quartier, situé dans une zone d'intense production immobilière et faisant face au nouveau Musée national de la Civilisation égyptienne - projet initié en son temps par Hosni Moubarak - se concrétise par la destruction de bicoques et par les visites répétées des autorités et d'ingénieurs venus mesurer les terrains et recenser la population. Pour autant, les habitants ne savent pas s'ils auront droit à un relogement, et encore moins où...

Explicitée ci-dessus, la marginalisation des travailleurs des déchets et des tanneries ne signifie pas qu'ils seraient tous, et de façon équivalente, confinés dans leurs espaces de vie et de travail et isolés du reste du monde. D'une part, cette assertion est relativisée par le fait que, à Manshiyyat Nâsir, les chiffonniers ont fait l'attention d'une forte attention des ONG, de programmes de coopération bilatérale et internationale et d'une médiatisation à l'étranger, notamment liée à I'activisme de Sour Emmanuelle (Du Roy, Furniss 2010). En ce sens ce vaste quartier se distingue des tanneries et des autres quartiers de chiffonniers méconnus. D'autre part, hormis pour les femmes, l'intégration économique au reste de la ville, impliquant des circulations des hommes et des matières, est essentielle au fonctionnement des activités. Nous y reviendrons après avoir 
explicité ci-dessous les façons d'entrer dans le travail, étroitement liées à la famille et à la communauté, ainsi que les parcours professionnels progressivement divergents de ces travailleurs.

\section{ITINÉRAIRES ET PRATIQUES PROFESSIONNELLES DES TRAVAILLEURS DES DÉCHETS ET DES TANNERIES}

\section{Être chiffonnier, une histoire de famille}

Au Caire, les chiffonniers le sont de père en fils et les trieuses de déchets, de mère en fille. Pourtant, les premiers d'entre eux, avant de migrer vers la capitale dans les années 1930-1940, étaient ouvriers agricoles ou métayers, rarement propriétaires de lopins de terre, même si tous dans une situation de grand dénuement, motif de leur exode. Si l'on ne sait pas, précisément, quelle est la première famille qui a décidé de "venir manger le pain au Caire », la reconstitution de plusieurs itinéraires permet de retracer leur histoire migratoire. II apparaît ainsi que beaucoup sont originaires de deux villages, Deir Tasa et el Badâri, au sud de la ville d'Assiût : dans ces bourgs, proches l'un de l'autre, les familles coptes sont liées par des relations fortes de parenté et d'interconnaissance.

Aussi, rares sont les arrivants qui tentent l'aventure migratoire seuls, sans connaître quelqu'un au Caire arrivé avant : un frère, un oncle, un cousin qui pourra les héberger et les introduire dans le monde du travail. II est certain que la proximité résidentielle de la famille élargie joue un rôle essentiel pour l'établissement de ces migrants. Une fois au Caire, ces hommes font venir le reste de leur famille; d'autres, comme le grand-père de 'Atef, organisent des allers-retours entre Le Caire et al-Badâri où sa famille demeure en attendant que la situation au Caire se stabilise; d'autres, encore, comme les grands-parents de Youssef décident de rompre définitivement leurs attaches à Deir Tasa et emmènent avec eux leur épouse et leurs enfants : ils effectuent un voyage de trois jours, en charrette, avec leurs affaires et quelques chèvres. Pour survivre au Caire, ils travaillent pour les récupérateurs d'alors, les wahiyya qui récoltaient depuis la fin du xix ${ }^{e}$ siècle les déchets revendus comme combustible aux hammams et aux fours des cuisines (Assaad 1987 :188). En raison de leur pauvreté, les zabbâlîn ne sont pas en position de négocier au mieux avec les wahiyya : ils proposent d'effectuer eux-mêmes la collecte des déchets, mais les wahiyya continueront à recevoir, de la main à la main, une petite somme de la part des habitants pour le service rendu. C'est ainsi que, rue par rue, quartier par quartier, chaque territoire de collecte se dessine, contrôlé par un wahi. Dans les quartiers les plus aisés, dont la valeur en déchets est plus intéressante, de nombreux zabbâlîn leur achèteront par la suite le droit de collecte. Cette organisation professionnelle est structurée - et ce encore actuellement - par le 'orf, à savoir la confiance, fondée sur la parole donnée (Assaad 1987 : 188), ce qui s'explique aussi par le fait que, à l'époque, wahiyya et zabbâlîn étaient pour la plupart 
analphabètes. Elle se transmet aussi de père en fils car les territoires de collecte sont héréditaires et les chiffonniers mettent un point d'honneur à ne pas empiéter sur le territoire de l'autre, car cela signifierait lui voler ses ressources.

L'origine commune, le voyage long et difficile, I'installation au Caire dans des bicoques sans cesse menacées parce qu'échafaudées sur des terrains squattés, le contexte d'incertitude et de grande misère forment un moment fondateur de l'histoire des chiffonniers, qui s'est transmis jusqu'à aujourd'hui. Cette histoire est encore à l'œuvre puisque de nombreux saisonniers de l'agriculture viennent de Haute-Égypte pour se faire embaucher ponctuellement dans les ateliers de recyclage et que d'autres retournent tous les trois mois au pays voir leur famille ainsi que l'explique Rizk, recycleur de plastique et l'un des leaders reconnus des zabbâlîn : "Les Sa'îdis sont des hard workers, ils font des allers et retours et travaillent beaucoup » (entretien du 6/11/2012). En 2017, Rizk nous explique que, depuis quelques années, certains de ces Sa'îdis, forts de leur expérience professionnelle ont ouvert des ateliers de recyclage en HauteÉgypte et ne reviennent plus au Caire (entretien du 19/02/2017). Cette référence aux Sa'îdis revient au fil des entretiens et marque la différence avec les citadins du Caire ou avec les fellahîn du delta. Sans donner un rôle trop important à cette distinction identitaire qui tend à s'estomper en raison de l'ancrage citadin, elle est utilisée, parfois avec humour, pour prouver la force, le courage et la virilité des Sa'îdis, que ce soit de la part des zabbâlîn ou de celle des travailleurs des Tanneries.

Enfin, bien que ce ne soit pas le seul élément constitutif de leur communauté professionnelle et, plus largement, de leur identité, il est certain que la dureté initiale de leurs conditions de vie et de travail a joué un rôle dans les représentations que les chiffonniers ont d'eux-mêmes, dans leurs stratégies d'évitement des autres citadins ainsi que, dans la mesure du possible, des pouvoirs publics.

\section{Venir travailler aux Tanneries : I'entremise des « oncles»}

Les filières migratoires vécues par les chiffonniers dans les années 1930-1940 sont assez similaires à celles des travailleurs ${ }^{25}$ des Tanneries dans les années 1950 à ceci près que, pour ces derniers, le projet professionnel est très précis : ils viennent au Caire parce qu'ils savent, dès avant leur départ, qu'ils pourront être embauchés dans les tanneries et cette stratégie professionnelle les motive avant tout. Ce savoir émane de voisins du village ou d'un membre de la famille qui y est déjà employé. En effet $\mathrm{si}$, dans la première moitié $d u x^{\mathrm{e}}$ siècle, les patrons des tanneries sont majoritairement Cairotes ou étrangers, ils emploient des hommes de Moyenne ou Haute-Égypte :

25 Il s'agit ici des travailleurs employés dans les tanneries et non des patrons. 
«Mon grand-père était tanneur en Grèce, mais ses affaires ne marchaient pas bien. Il est parti à Jaffa, en Palestine, en 1887, puis il est venu ici en 1948 à cause de la guerre (...). Mon père et ses cinq frères ont ensuite loué une tannerie à un Égyptien, car à cette époque, les étrangers ne pouvaient pas être indépendants. Puis, chacun a eu sa tannerie ainsi que mes cousins (...). Au début, on avait 12 ou 13 ouvriers qui venaient de Haute-Égypte parce que ce travail est dur et que seuls les gens du Sa'îd sont assez forts pour supporter ce travail ${ }^{26}$ (entretien avec Youssef Kiriasi, patron d'une grande tannerie, 17/10/1995).

La stratégie résidentielle de ces premiers travailleurs n'est pas vraiment anticipée et plutôt que de s'installer dans ce lieu vide et répulsif, ils vont être accueillis temporairement par des proches, louer des petites pièces dans des quartiers populaires voisins ou être hébergés dans les tanneries mêmes. Pour certains, il s'agit d'une migration directe et définitive, mais d'autres effectuent des migrations saisonnières, en fonction des récoltes quelques années durant, avant leur ancrage au Caire. Ce sont des adolescents ou hommes jeunes qui ont laissé femmes et enfants au village. Beaucoup sont originaires de Sifilâq et de Sawâm'a el Charq, deux bourgs de la région du Sohag, ou encore de la région du Fayyûm où ils ne pensent avoir aucun avenir en raison de la pauvreté.

À partir des années 1950-1960, alors que l'activité de tannage se développe et que les habitations informelles se multiplient, se met en place une filière migratoire professionnelle pour deux raisons. D'une part, cet espace, plus peuplé leur semble moins hostile et le quartier s'édifie progressivement. En découle un effet d'entraînement et d'accélération de son peuplement et ce n'est plus par hasard qu'on s'y installe, mais bien parce que l'on y connaît déjà quelqu'un qui y vit. 'Izbat al-Madabegh constitue alors un point de chute, projeté et définitif au Caire. À partir du moment où l'habitat se consolide, les épouses rejoignent leur mari. Les alliances consanguines, très fréquentes, participent aussi à l'arrivée dans le quartier de jeunes femmes qui s'y marient et fondent un foyer avec un cousin installé depuis plus longtemps.

D'autre part, à partir de cette décennie, les petites tanneries et, surtout, les ateliers de glu et gélatine, dont la fabrication est artisanale et ne demande que peu d'investissement, se multiplient. Il s'agit de petites entreprises familiales dont le mode d'embauche aide les derniers venus à " grimper » plus rapidement que leurs prédécesseurs dans la hiérarchie des métiers du cuir et même à accéder à l'indépendance professionnelle, parce qu'ils ont acquis dans ces petites fabriques une expérience professionnelle. Pour les jeunes hommes, la

26 J'ai entendu ce propos sur la «force des Sa'îdis » à de nombreuses reprises. On peut surtout penser que dans un contexte migratoire tendu, les migrants de HauteÉgypte, plus démunis que d'autres, n'ont pas vraiment le choix du travail et sont affectés aux tâches les plus difficiles. 
présence d'un " oncle ${ }^{27}$ ou protecteur plus âgé propriétaire d'une petite tannerie (sous-traitante d'une grande) ou d'une fabrique de gélatine, ou encore celle d'un frère ou d'un cousin qui a pu commencer comme porteur de peaux ${ }^{28}$ mais qui obtient ensuite un emploi plus stable, jouent un rôle fondamental quant à leurs itinéraires professionnels et résidentiels. De façon plus générale, lorsque la position professionnelle du membre de la famille déjà sur place est subalterne et précaire, l'entraide familiale joue un rôle plus limité dans le processus d'installation des nouveaux venus. Alors que, à l'inverse, les oncles tanneurs, frères ou cousins « éclaireurs » qui ont " grimpé » dans le métier sont des relais essentiels car la transmission des expériences, en particulier professionnelles, passe aussi par eux.

L'ancrage résidentiel est dès lors plus facile pour ces migrants car ils connaissent les modalités d'accès au sol et sont aidés lors de la construction de leur habitation. Dans les années 1980, I'indépendance professionnelle d'une partie d'entre eux se traduit par une amélioration des conditions de logement : certaines bicoques sont remplacées par un habitat à étages qui accueille de nouveaux membres de la famille ou qui permet d'héberger un fils marié. Cette cohabitation familiale intergénérationnelle permet de « travailler en famille » et garantit ainsi la subsistance de tous.

\section{LES DISTINCTIONS PROFESSIONNELLES : PATRONS TANNEURS ET «BUSINESSMEN DE LA PROPRETÉ »}

Deux générations de patrons de tanneries se distinguent : les plus anciens, natifs du Caire ou de l'étranger, qui ont implanté leurs fabriques avant les années 1950 et les plus récents, migrants du Sa'îd, qui ont pu, grâce à leur ascension professionnelle dans le métier, ouvrir des ateliers. Ceci dit, les uns et les autres sont étroitement liés. L'itinéraire d'Ernest Salem Zarifa témoigne de ces relations. En 1919, le grand-père d'Ernest, Henari Salem Zarifa et ses deux fils Yuraki (père d'Ernest) et Gabra, arabes chrétiens de Gaza, construisent une première tannerie, rue Salem Zarifa qui hérite du patronyme familial. En 1940, Yuraki ouvre une seconde tannerie rue Fûstat dans laquelle il travaille avec ses six fils qui, devenus adultes, seront aussi propriétaires de tanneries. De son côté, Gabra se sépare de son frère et ouvre deux nouvelles tanneries, rue Sikkat el Hadid, où il fait travailler 150 personnes :

27 Le mot " oncle » peut renvoyer au lien de parenté mais, plus largement, à une personne plus âgée.

28 À l'époque, il s'agit des charretiers qui transportaient les peaux des abattoirs de Sayyida Zaynab jusqu'aux tanneries, puis qui transportent les peaux tannées des tanneries jusqu'aux ateliers de confection de la vieille ville. 
«Gabra, c'était un grand tanneur, il fabriquait du cuir de mouton, de vache, de chameau, des semelles et du cuir pour les chaussures et les vêtements (...) L'un de ses enfants, Michel, fabriquait et vendait des sacs en cuir dans le Khan al-Khalili (...) tout le reste de la famille travaillait dans les tanneries ! (Entretien avec Ernest, $11 / 11 / 1995)$.

En 1963, certaines grandes tanneries ainsi que celles appartenant à ces étrangers sont nationalisées : c'est le cas pour Gabra Salem Zarifa, pour le khawaga ${ }^{29}$ Yanni (Arménien), pour le khawaga Cristofedes ou le khawaga Kostayyani (Grecs), ou encore le khawaga Starro Kiriasi (Grec ayant migré en Palestine puis en Égypte) et de nombreux autres, ce qui n'arrête pas pour autant leur activité de tanneurs. Ernest, âgé de 74 ans lorsque nous le rencontrons en 1995, est un homme élégant et cordial qui s'exprime en très bon français, ce qui prouve son niveau d'éducation :

« Dans le temps, ce métier était un très bon métier, aujourd'hui c'est un métier de merde! C'est devenu catastrophique lorsque les petits tanneurs se sont installés. Ils sont arrivés comme des parasites. Les ouvriers qui travaillaient chez moi sont partis pour être des indépendants, mais sans avoir la technique ni les moyens. »

L'un des travailleurs d'Ernest était Hamdi qui revendique au contraire ses compétences professionnelles, même si son activité n'est pas formalisée :

« Mon frère aîné est arrivé en premier [vers 1955] et a commencé par travailler dans la tannerie de Gabra Salem Zarifa, puis chez Kiriasi. Les Grecs travaillaient bien! Moi, j'ai commencé ici à 17 ans avec mes trois autres frères. Je mettais les peaux dans les barils, avec les produits chimiques, je connaissais toutes les étapes du travail. En 1964, j'ai acheté une petite tannerie, puis je l'ai agrandie (...). J'aime ce travail, parce que j'ai passé ma vie à le faire ! » (entretien avec Hamdi 30/08/1996).

En 2017, cette représentation différenciée entre anciens et nouveaux tanneurs perdure ainsi que l'explique l'ingénieur d'une grosse tannerie qui a pignon sur rue :

29 Khawaga signifie étranger et c'est ainsi que ces tanneurs sont recensés dans les archives de la Chambre des Tanneurs, datant de 1938 et que j'ai consulté en 1996. 


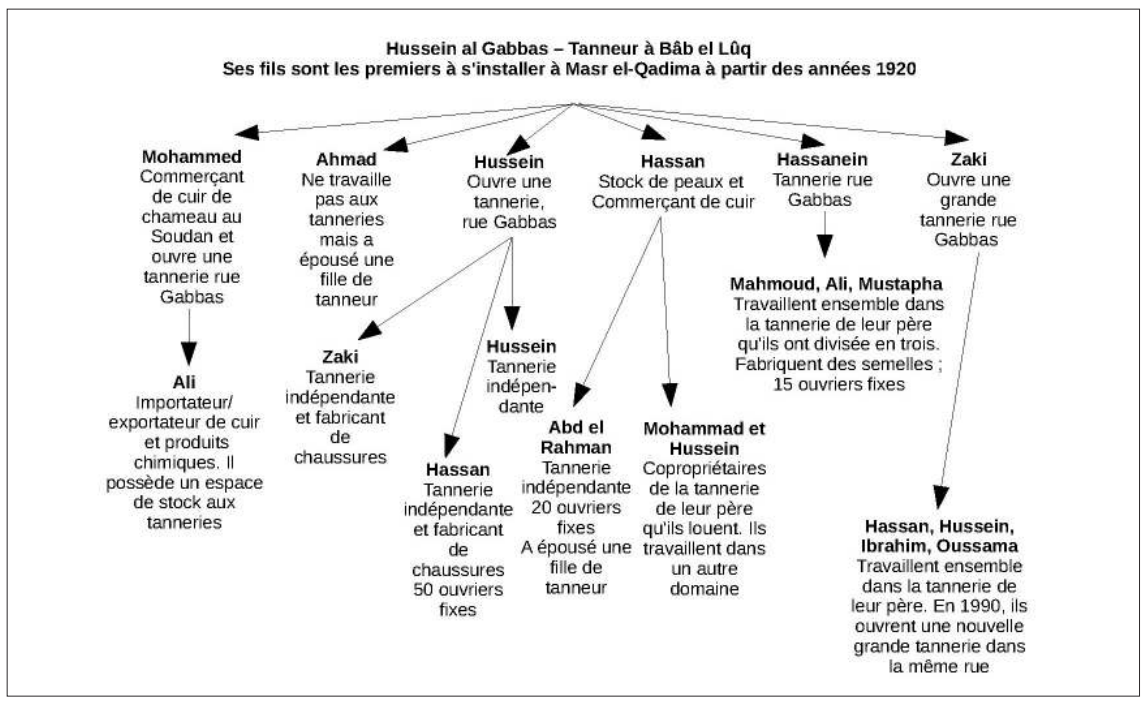

Figure 7 : Généalogie simplifiée des tanneurs de la famille Gabbas (les prénoms ont été changés et nous n'avons pas indiqué les femmes ni les enfants employés dans d'autres activités). La famille Gabbas est I'une des plus anciennes et connues des Tanneries, notamment par son activité de tannage des peaux de chameaux provenant de Haute-Égypte, à l'origine livrées par bateau au Caire. La généalogie de cette famille cairote montre les activités des descendants de Hussein al Gabbas qui se diversifient tout en restant dans les métiers du cuir ou apparentés. L'installation précoce dans le quartier a permis à la famille d'asseoir sa position sociale et professionnelle : elle est ainsi représentée à la Chambre des Tanneurs, a occupé de nombreux terrains pour étendre ses activités tout en étant favorable au projet de délocalisation voulu par les autorités (sources : B. Florin, entretiens avec Hassan al-Gabbas et Abd al-Rahman)

«Le mot «ma'allem», c'est un mot «baladi» [populaire]; les patrons des grandes tanneries, on les appelle plutôt «rayyîs» ou «sâhib el Madabegh» ${ }^{30}$, car ce sont des hommes d'affaires qui ont investi dans des techniques et des machines modernes. Par contre, les fabricants de glu et gélatine ou les indépendants [informels, non-inscrits à la Chambre], sont restés des «ma'allemîn»! » (entretien du 23/02/2017).

Au sein des activités de tannage formelles, la hiérarchie est forte entre les porteurs de peaux, les travailleurs spécialisés, ceux qui manipulent les produits, les contremaîtres, etc. S'ajoutent les groupes précis qui gravitent autour des bâtiments tels les charretiers indépendants qui se regroupent à des endroits précis du quartier, par exemple le long de l'Aqueduc où les tanneurs savent les

30 Ma'allem (plur. Ma'allemîn) signifie « chef » artisan, patron d'atelier, patron d'un café populaire, etc.; rayyîs (raïs en français) rend compte d'un titre et d'une position beaucoup plus importante qui désignait un dignitaire ottoman, et aujourd'hui un chef d'État (Nasser avait pris le titre de raïs), sâhib signifie propriétaire. 
trouver ou encore les «clouteurs » de peaux dont I'unique tâche consiste à tendre puis clouter les peaux sur des cadres en bois qui sèchent au soleil, rue Fustât. L'on peut encore évoquer les gâladîn, commerçants de peaux achetées dans les abattoirs et les campagnes et revendues aux tanneries.

Cette distinction professionnelle se retrouve, sous une autre forme, chez les chiffonniers : depuis les années 1980 et surtout 1990, la mise en œuvre de nouvelles techniques de recyclage et leur ingéniosité, I'adaptabilité des activités en fonction des matériaux récupérés, les savoir-faire en termes de négociation et commercialisation, I'insertion progressive dans des réseaux industriels rendent compte de la professionnalisation de ceux qui, enfants, récupéraient la nourriture pour leurs animaux et qui, aujourd'hui, sont devenus des patrons influents $d^{\prime}$ un système que personne n'a vraiment organisé. L'ascension professionnelle n'est pas généralisée, mais elle est devenue possible ainsi que le montre la généalogie de la famille de Youssef ci-dessous.

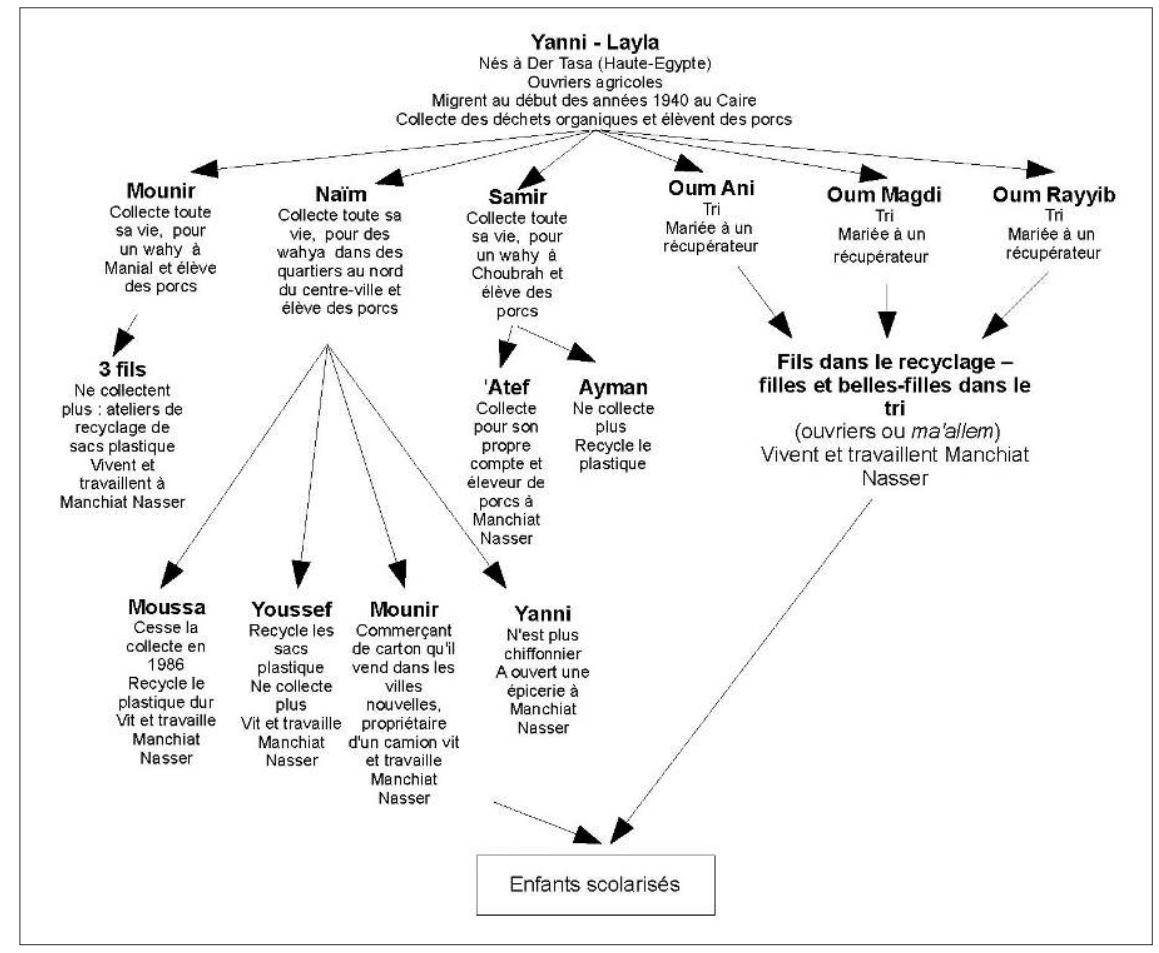

Figure 8 : Généalogie simplifiée de la famille de Youssef (zabbal), (les prénoms ont été changés). La troisième génération de cette famille installée depuis les années 1940 n’est plus affectée à la collecte des déchets en ville, mais détient des ateliers et des machines-outils pour le recyclage du plastique. Malheureusement, nous n'avons pas d'informations très précises sur les trajectoires des femmes de la famille qui travaillent pour leurs époux et qui sont (ou ont été par le passé) affectées au tri des déchets. Cette affectation est donc à prendre avec prudence dans le sens où les épouses de patrons d'ateliers générant de confortables revenus cessent l'activité de tri (sources : B. Florin, entretien avec Youssef, février 2014) 
Elle se manifeste dans toutes sortes de domaines : les efforts consentis pour la scolarisation des enfants, l'amélioration de l'habitat et la construction de nouveaux immeubles, I'autonomisation par rapport à la famille, ou encore l'émergence de leaders qui ont porté les revendications d'un droit au travail et qui se sont manifestées publiquement dans les années 2000 contre les réformes en cours (Florin 2011 et 2015).

La hiérarchie qui différencie les tanneurs se retrouve dans la distinction professionnelle également présente chez les zabbâlîn qui forment une corporation hétérogène. Cette hiérarchie est paradoxale : ainsi, les éleveurs de cochons du quartier de Manshiyyat Nâsir, loin d'être les plus pauvres, sont cependant considérés comme faisant un travail ingrat, bien que nécessaire à tous et d'ailleurs de nombreux recycleurs ont eux-mêmes des cochons, à l'instar de l'un des fondeurs de canettes d'aluminium de Manshiyyat Nâsir, activité très rentable qui le positionne au sommet de la hiérarchie. La professionnalisation de certains explique qu'il est difficile aujourd'hui de les qualifier de zabbâlîn : «Nous ne sommes pas des éboueurs [...] mais des businessmen des ordures! » (Romani ${ }^{31}$, recycleur à Manshiyyat Nâsir, entretien du 09/02/2010). Lise Debout note également que l'expression â'mâl nazâfa signifie littéralement "businessman de la propreté », expression utilisée par les chiffonniers aisés pour se désigner autrement que par zabbâlîn qui a, pour eux, une connotation négative (Debout, 2015 : 427).

Quoi qu'il en soit, la hiérarchie professionnelle est étroitement liée à la valeur des matériaux transformés qui se décline depuis le sommet par le recyclage du métal (minoritaire), puis celui du plastique (majoritaire), puis celui des cartons, puis par les papiers et chiffons. Bien sûr, il faut y ajouter l'élevage des porcs, véritable " capital sur pattes », dont le lisier ainsi que la viande se vendaient très bien avant leur abattage en $2009^{32}$.

\section{UN ARTISANAT INDUSTRIEL QUI REPOSE SUR LE SECTEUR INFORMEL}

Qu'il s'agisse des tanneurs ou chiffonniers, la hiérarchie professionnelle (et sociale) est visible autant par la nature des activités que par le biais des outils de production utilisés. Elle distingue les activités restées artisanales de celles qui s'apparentent davantage à un processus industriel. En effet, certaines activités peuvent être très lucratives tout en restant très artisanales comme $c^{\prime}$ est le cas pour la production de lingots d'aluminium évoquée ci-dessus. À Manshiyyat Nâsir, le ma'allem Barakat est l'un des trois recycleurs d'aluminium du quartier. Il dispose d'un four qui fond à 660 degrés les canettes. Quatre ou cinq travailleurs y travaillent à l'aide de casseroles à long manche qu'ils remplissent de

31 Romani avait déjà employé cette expression avant cet entretien (Dollet, 2003 : 98). 32 Voir l'Introduction générale de cette livraison ainsi que Florin 2011 et Tadroz 2010. 


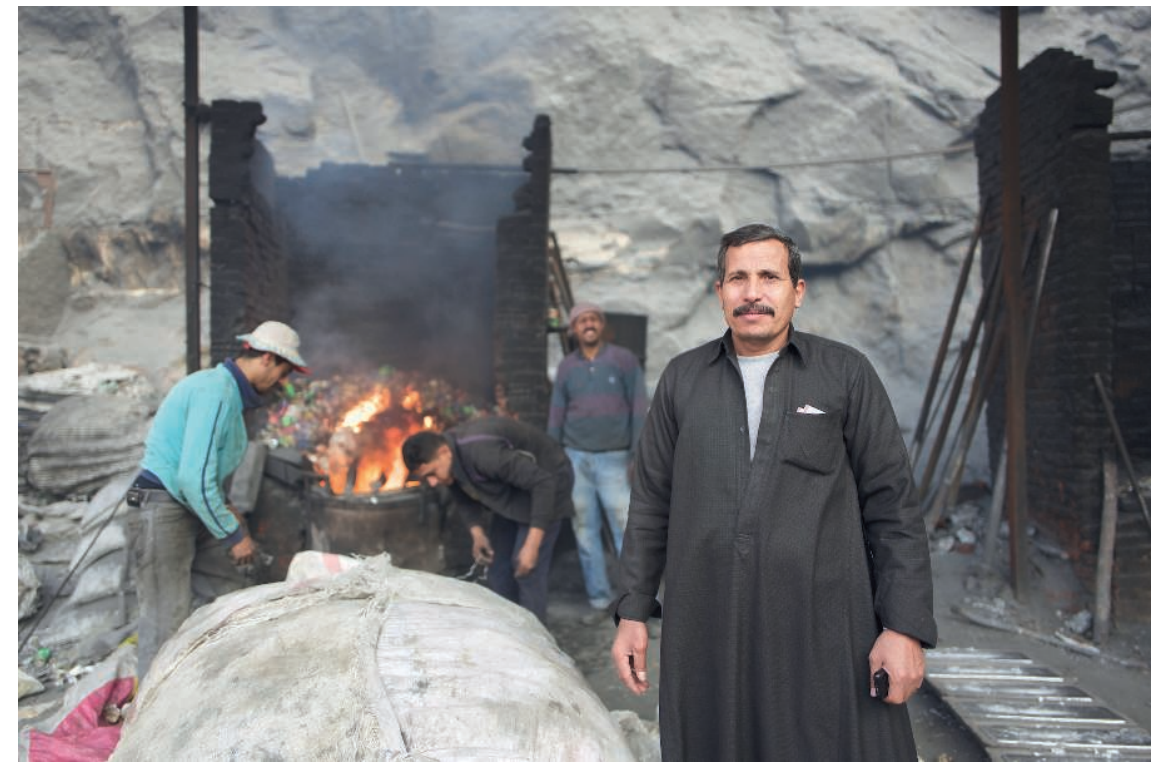

Figure 9 : Les travailleurs de Barakat (2017). Le ma'allem Barakat avec ses ouvriers fondeurs d'aluminium (photo Pascal Garret, Le Caire, 2017)

métal fondu versé dans des moules en ciment réfractaire, puis démoulé à l'aide de pinces. Le procédé est plus que simple pour une opération très rentable même si les vapeurs qui s'en dégagent sont hautement toxiques.

Les techniques de recyclage des papiers et cartons ou du plastique sont inégalement perfectionnées d'un atelier à l'autre : ainsi, Romani a fabriqué lui-même, avec des métaux de récupération, un broyeur de plastique, mais depuis 2000 et surtout 2010, une dizaine de patrons d'ateliers a pu acquérir des machines importées de Chine, dont le prix se situe aux alentours de $100000 €$ (Entretien avec Romani, 25/02/2017). À la suite de la révolution de 2011 - qui a laissé le champ libre à une intense activité de construction - se sont ouverts de grands ateliers pour le compactage des papiers et cartons qui nécessitent de grandes surfaces. Ces capacités d'investissement dans des locaux, des machines-outils, des véhicules, même si réservés à une minorité, tendent à transformer leurs activités en un artisanat industriel qui se caractérise aussi par une forte intégration dans des réseaux de commercialisation avec les industries formelles égyptiennes mais aussi étrangères : les grossistes en ville achètent des objets fabriqués à Manshiyyat Nâsir (cintres en plastique noir, seaux, etc.); les cartons compactés et lingots d'aluminium sont vendus aux usines du secteur formel; les boulettes de plastique ou palettes de bouteilles compressées sont destinées aux usines détenues par des Chinois dans les villes nouvelles du Grand Caire ou même exportées en Chine; les accords se multiplient entre chiffonniers des villes de province ou des sites touristiques et patrons d'ateliers de Manshiyyat Nâsir pour valoriser les déchets dans la 


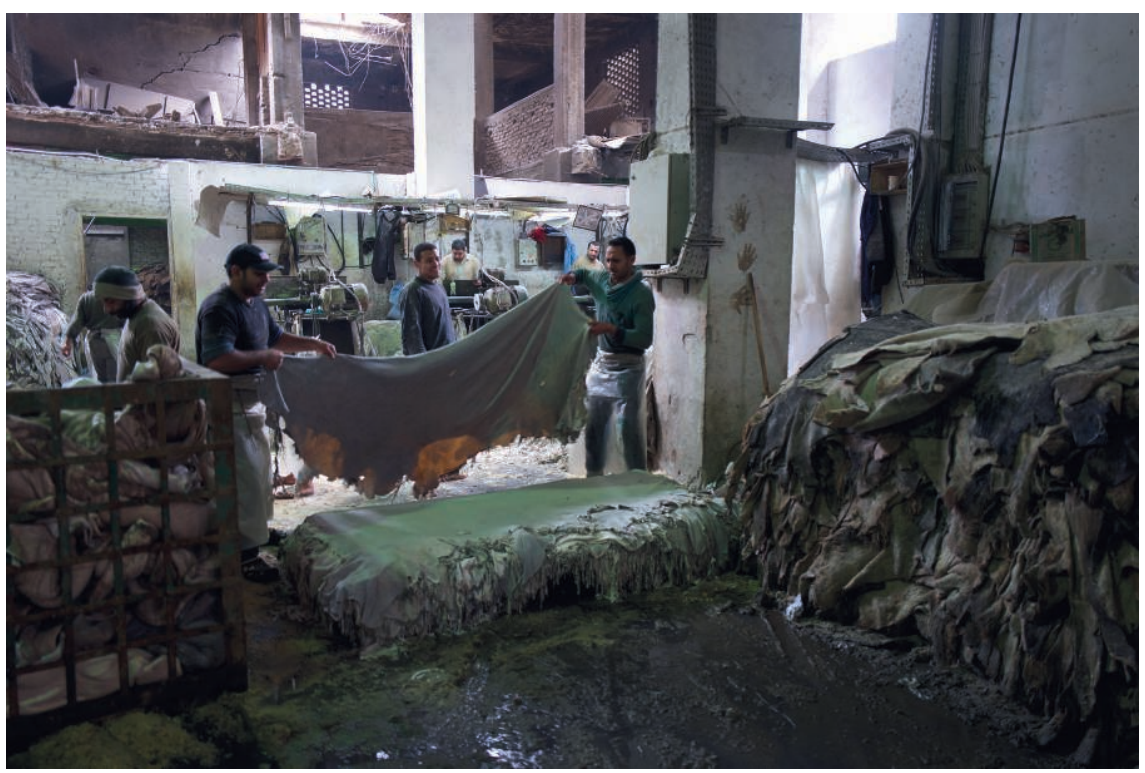

Figure 10 : Le travail dans une grande tannerie. Travailleurs d'une grande tannerie formelle, rue Fustât (photo Pascal Garret, Le Caire, 2017)

capitale. Cette dynamique économique et ces nombreux liens avec le secteur formel ne doivent pas masquer les inégalités professionnelles dans et entre les quartiers de chiffonniers et, notamment, à la base de l'échelle professionnelle, les récupérateurs à pied ou en charrette et les femmes qui ont des conditions de travail plus que difficiles. De même, l'activité des ateliers et le rôle des commerçants de Manshiyyat Nâsir ont placé ce grand quartier de quelque 60000 chiffonniers dans une position dominante par rapport aux autres, encore renforcée par les interventions développementalistes évoquées plus haut. L'importance de la main-d'œuvre, le nombre de camions, pick-up, machines, ateliers, etc., mais aussi la nature des relations avec le secteur formel renforcent la dépendance des autres petits quartiers de chiffonniers à Manshiyyat Nâsir, provoquant I'amertume de certains comme à Batn el-Baqara.

Enfin, dans le cas des tanneries, ces divergences professionnelles distinguent les travailleurs indépendants, payés à la tâche et ne bénéficiant d'aucune protection sociale, assurance maladie ou retraite, contrairement aux travailleurs salariés fixes. Les « indépendants » sont aujourd'hui beaucoup plus nombreux que les «fixes » qui travaillent dans les grandes tanneries où ils sont attachés à un patron. Dans ces dernières, la modernisation des techniques et des processus de production en un peu plus de vingt ans est évidente : s'il reste toujours de nombreux vieux barramil en bois qui furent fabriquées par des menuisiers locaux, les barramil en métal, qui n'existaient pas en 1995, se sont multipliés ainsi que des machines-outils modernes et importées pour étirer et affiner les peaux. 
En même temps, ces activités formelles/informelles sont loin d'être étanches car les premières sous-traitent aux secondes le travail mais aussi la matière qui circule : les résidus organiques des peaux sont vendus aux fabricants de glu et gélatine, de même que la laine aux fabricants de bourre; de nombreuses tâches manuelles existent toujours comme le cloutage des peaux sur les cadres de bois, le portage des peaux d'un lieu à l'autre à dos d'homme ou sur les charrettes, la délégation d'une partie de la production à des " petits » tanneurs qui réalisent seulement une étape du tannage, etc. Même les poils des queues des animaux sont récupérés et deviendront des pinceaux... On peut encore citer ici les intermédiaires que sont les galadîn, - dérivé de " geld », le cuir - corporation héréditaire de commerçants ${ }^{33}$ qui apportent aux tanneries du Caire les peaux achetées dans les abattoirs égyptiens ou dans les campagnes, peaux vendues ensuite à des simsars - courtiers - qui, eux, les stockent avant de les revendre avec une commission de $7 \%$ aux tanneries. Sans oublier ici les vendeurs de produits chimiques ou encore ceux qui récupèrent les chutes de cuir pour en faire des ceintures ou des lanières de sandales. Certes, certains métiers ont disparu entre 1995 et 2017 comme les « racleurs » qui, grâce à une grande lame courbe munie de deux poignées décrochaient manuellement les résidus organiques des peaux pour la gélatine : «On est payé 70 piastres par peau et ça nous demande $1 / 4$ d'heure de travail pour chacune. Je gagne entre 35 et 40 livres $^{34}$ égyptiennes par jour (...). Seulement cinq hommes font encore ce métier car maintenant c'est la machine qui nous remplace » (Hassan Osman, entretien du 30/07/1995). De même, le travail des hommes qui malaxaient les peaux, à jambes nues dans l'eau chargée de produits chimiques des lavoirs, a disparu aujourd'hui.

Quoi qu'il en soit, c'est bien cette synergie et souplesse organisationnelles entre activités formelles et informelles qui est remise en cause par la délocalisation des tanneries.

\section{CONCLUSION}

La mixité fonctionnelle, articulant petites activités économiques non industrielles et logements auto-construits, a construit les paysages urbains singuliers au Caire que sont les Tanneries et les quartiers des chiffonniers. Proposé ici, le rapprochement entre les unes et les autres se justifie aussi par les histoires de vie de leurs habitants-travailleurs : les migrations de Haute-Égypte, I'installation

33 Depuis 1994, les galadîn les plus importants font partie de la Chambre des tanneurs, sur demande de celle-ci afin de pouvoir contrôler les prix, mais la plupart sont informels.

34 Un peu moins de 10 euros pour l'époque, ce qui représentait alors un salaire correct. 
au Caire, l'édification lente et laborieuse des habitations, I'entrée dans le travail et I'ascension professionnelle de certains constituent autant de convergences. Ceci n'annule pas les différences telles que, par exemple, I'ancienneté de la présence des patrons tanneurs face à la relative jeunesse des entrepreneurs des déchets, ou encore les contrastes entre les différents quartiers de chiffonniers. L'imbrication, dans chacun des cas entre activités formelles et activités informelles est très forte : pour les Tanneries, elle se réalise au sein même du quartier, par le biais de la sous-traitance des travailleurs indépendants payés à la tâche par les tanneurs inscrits à la Chambre des Tanneries. Pour les chiffonniers, majoritairement informels, elle se réalise hors du quartier grâce à leurs relations avec le secteur du recyclage formel local ou international ${ }^{35}$.

Si l'inclusion des activités informelles dans les activités formelles est évidente, ce clivage garde un sens pour les acteurs : à Manshiyyat Nâsir, les entretiens menés depuis 2007 révèlent un refus systématique de toute formalisation des ateliers qui coûterait trop cher en taxes et mise aux normes. Aux Tanneries, la posture des protagonistes oppose les fabriques formelles, appartenant à la Chambre, aux fabriques informelles dont les patrons savent qu'ils n'auront pas droit à la délocalisation, ni en termes financiers, ni en place prévue pour eux. Au-delà de ce clivage, cette délocalisation cristallise les tensions depuis 30 ans entre les propriétaires des vieilles tanneries qui n'ont jamais eu les moyens de se moderniser, dont les baramil en bois sont inamovibles, aux « grands tanneurs » qui pourront déplacer sur le nouveau site leurs machines-outils modernes et qui, d'ailleurs, approuvent fermement le déplacement à Badr City. Quant aux travailleurs de ces mêmes tanneries, habitant avec leurs familles dans le quartier ou à proximité, ils sont très inquiets de ce déplacement à $80 \mathrm{~km}$ du Caire, dans une ville nouvelle où ils pourront sans doute obtenir des appartements mais où ils ne connaissent personne, où le logement avec la famille élargie est impossible, où ils ne disposeront pas de moyens de transport, etc. Et qu'en sera-t-il pour tous les travailleurs informels des activités annexes? Ces derniers disent qu'ils devront se reconvertir, mais un fort sentiment $d^{\prime}$ 'injustice, de colère et d'amertume les anime. De façon plus générale, et quels que soient les statuts et positions de ces travailleurs ou patrons, ce sont aussi les liens communautaires noués de génération en

35 De la même façon, les grands tanneurs sont intégrés dans des réseaux internationaux : I'étude des circulations du cuir montre des importations de peaux brutes depuis I'Europe (où elles ne peuvent être traitées en raison des normes anti-pollution) jusqu'aux tanneries du Caire qui traitent puis ré-exportent ces peaux vers l'Italie et I'Allemagne pour la confection de produits en cuir de qualité ou vers la Chine pour la qualité moindre, produits qui seront ensuite revendus en Europe, parfois par les mêmes commanditaires initiaux. On notera ici l'exportation de ces activités polluantes européennes vers l'Égypte. 
génération et ce depuis presque un siècle, au sein de ce quartier, qui risquent de se déliter.

Enfin, appartenant à la ville-centre où les prix du foncier sont très élevés, ces espaces de vie et de travail font depuis longtemps l'objet de convoitises des promoteurs immobiliers et de projets de réaménagement soutenus par les acteurs politiques ${ }^{36}$. Certains ont déjà disparu, à l'instar du quartier des potiers démoli vers 1994, d'autres sont plus que menacés comme à Batn al-Baqara où les premières démolitions d'habitations de chiffonniers ont déjà été effectuées. Quant aux Tanneries du Caire, elles ne seront sans doute plus qu'un souvenir d'ici peu... Si la nature polluante de ces activités peut à juste titre justifier leur déplacement, il n'en reste pas moins que les modalités choisies pour le réaliser interrogent sur les effets économiques et sociaux qui concerneront la majorité de ces travailleurs et la disparition d'un patrimoine industriel et artisanal de plus d'un siècle.

\section{BIBLIOGRAPHIE}

Assaad R., 1987, "L'informel structuré. Les zabbâlin du Caire », Peuples méditerranéens, $n^{\circ} 41-42$, p. 181-192.

Cirelli C. Florin B., 2015 «Vivre des déchets », in C. Cirelli et B. Florin (dir.), Sociétés urbaines et déchets. Une comparaison internationale, Tours, Presses Universitaires François Rabelais, p. 13-56.

Clot-Bey A-B., 1840, Aperçu général sur l'Égypte, Fortin, Masson et Cie, Paris.

Corteel S. Le Lay S., 2011, Les travailleurs des déchets, Paris, Éditions Erès.

Debout L., 2015, "Lexique», in C. Cirelli et B. Florin (dir.), Sociétés urbaines et déchets, Tours, Presses Universitaires François Rabelais.

Debout L. Florin B, 2011, "Les contradictions du nouveau système de déchets au Caire. Conflits, négociations et stratégies d'acteurs ", Égypte-Monde arabe, 3ème Série, n 8 , p. 31-57.

Desvaux P. Furniss J, 2015, "Hybridations formelles informelles : gestion des déchets au Caire après la délégation à des entreprises internationales », in K. Bennafla (dir.), Acteurs et pouvoirs dans les villes du Maghreb et du Moyen-Orient, Paris, Karthala, p. 219-238.

Dollet S., 2003, Une communauté traditionnelle face à la modernité. Le cas des zabbâlîn du Caire, mémoire de DEA en Sciences Politiques, sous la direction d'Élisabeth Picard, Université d'Aix-Marseille 3.

36 Voir par exemple la commande en 1995 d'une étude de délocalisation des tanneries conçue dans le cadre d'accords de coopération entre l'Institut d'Aménagement et d'urbanisme de la région Île-de-France. Aujourd'hui, le projet consisterait à transformer cet espace en "zone touristique » du fait de I'Aqueduc (sans que I'on ait davantage de précisions du contenu du projet). 
Du Roy G. Furniss J., 2010, « Sœur Emmanuelle et les chiffonniers : partage de vie et développement, 1971-1982 », in C. Sappia et O. Servais (dir.), Mission et engagement politique après 1945. Afrique, Amérique Latine, Europe, Paris, Karthala, p. 87-101.

Florin B., 1999, Itinéraires citadins au Caire : mobilités et territorialités dans une métropole $d u$ monde arabe, thèse de doctorat en Géographie, sous la direction de Pierre Signoles, Université François Rabelais, Tours.

Florin B., 2011, « Résister, s'adapter ou disparaître : la corporation des chiffonniers du Caire en question », in Y. Bonny et al. (dir.), Les Travailleurs du déchet, Paris, Erès, pp. 69-91.

Florin B., 2011, "Les chiffonniers du Caire et la grippe porcine », Les blogs du Diplo. Visions cartographiques. URL : https://blog.mondediplo.net/2011-01-12-Les-chiffonniers-du-Caire -et-la-grippe-porcine, consulté le 8/10/2018.

Florin B., 2015, "Les chiffonniers du Caire : soutiers de la ville ou " businessmen des ordures »? », Ethnologie française, vol. 153, pp. 487-498.

Florin B., 2015, "Quand la question des déchets devient un problème public : réforme, crise, ajustements et malentendus entre acteurs. L'exemple du Caire (Égypte) », in Cirelli C. et Florin B., Sociétés urbaines et déchets. Une comparaison internationale, Tours, Presses Universitaires François Rabelais, pp. 239-268.

Hugues E-C., 1962, « Good People and Dirty Work », Social Problems, vol. 10, n 1, p. 3-11. Ilbert R., 1989, "Égypte 1900 : habitat populaire, société coloniale », État, villes et mouvements sociaux au Maghreb et au Moyen-Orient, Paris, L'Harmattan, p. 266- 282.

Levi G., 1985, Le pouvoir au village. Histoire d'un exorciste dans le Piémont du xvII siècle, Paris, Gallimard.

Lhuilier D., 2005, "Le sale boulot », Travailler, n 14, n² 2, p. 73-98.

Mendels F., 1981, Industrialization and Population Pressure in XVIIIth Century Flanders, New York, Arno Press.

Raymond A., 1990, "Le déplacement des tanneries à Alep, au Caire et à Tunis à l'époque ottomane : un indicateur de croissance urbaine ", Revue d'histoire maghrébine, $\mathrm{n}^{\circ}$ 7-8, pp. 192-200.

Raymond A., 1993, Le Caire, Paris, Fayard.

Raymond A., 1993, "Les transformations des corporations de métiers au Caire du XVIII au xIx ${ }^{\mathrm{e}}$ siècle », in $\mathrm{H}$. Bleuchot, Les institutions traditionnelles dans le monde arabe, Paris, Khartala, p. 29-40.

Roussillon A., 1996, " "Comme si la ville était divisée en deux". Un regard réformiste sur I'urbain en Égypte au tournant des années 1940 », Genèses, vol. 22, nº 1, p.18-39.

Tadros M., 2010, «Scapepigging: H1N1 Influenza in Egypt », in S. Dry et M. Leach (dir.), Epidemics: Pathways of disease and response, London, Earthscan, p. 213-238. 